\title{
PROPOSED MODEL FOR ASSESSING THE GATED COMMUNITIES IN EGYPT USING FUZZY DELPHI EVALUATION MODEL
}

\author{
Ayman M. Zakaria Eraqi ,Aliaa El-saeid k. El-mallah \\ Architecture Dept., Faculty of fine arts, University of Minia, Minia, Egypt \\ *Corresponding author Email: Ayman.ibrahim@mu.edu.eg
}

\begin{abstract}
: -
Gated communities are one of the patterns that emerged with the beginning of the sixties, to achieve security, safety and uniqueness of some elite classes, and then later moved to become a kind of communities that achieves distinction for some individuals with the highest income class in society. And those communities emerged in the nineties in Egypt with the economic transformations of the pattern of free economy. The research conducted a study of the reasons for the selection of users of these communities, the reasons for their transition to and the requirements for satisfaction with them. The aim is to develop a model for assessing the success of the gated community and analyzing the weaknesses within it. To this end, the research used Fuzzy Delphi method (FDM), to deal with problems of verbal assessments, criteria overlaps and complexities, and confirm model building information. The main criteria for the model consisted of urban characteristics, economic characteristics, social characteristics, environmental characteristics, and administrative characteristics. The Fuzzy Delphi Evaluation Model (FDEM) was applied to the Al-Rehab neighborhood in Cairo as a case study, and the results showed an average success for the neighborhood in meeting the requirements of residents, achieving a sense of security and safety at a low rate, achieving a distinctive lifestyle, and population satisfaction, at an average rate.
\end{abstract}

Keywords: Gated communities - Fuzzy Delphi method - Fuzzy Delphi Evaluation Model - Egypt.

Received:21 January, 2020, Accepted: 5 February, 2020 
Vol. 40, No. 1. January 2021

نموذج مقترح لتقييم المجتمعات المسورة بمصر باستخدام طريقة دلفي الضبابية (FDM)

$$
\begin{aligned}
& \text { ايمن محمد زكرياعبد الوهاب" علياء سعيد كامل الملاحج }
\end{aligned}
$$

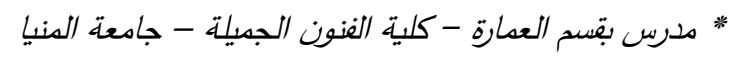

Ayman.ibrahim@mu.edu.eg

ملخص:

المجتمعات المسورة هي احد الانماط التي ظهرت مع بدايات الستينيات من القرن الماضي، لتحقيق الامن والسلامة والتثرد لبعض طبقات النخبة، ثم انتقلت لاحقا لتصبح نوعا من المجتمعات التي تحقق التميز لبعض افراد الطبقة الاعلى دخلا في ملي

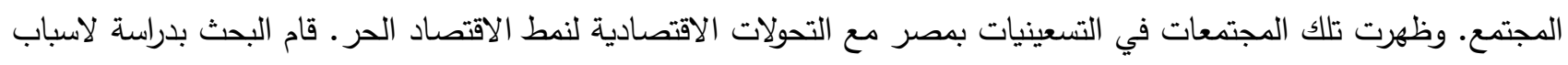

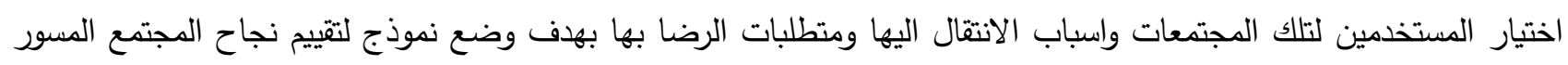

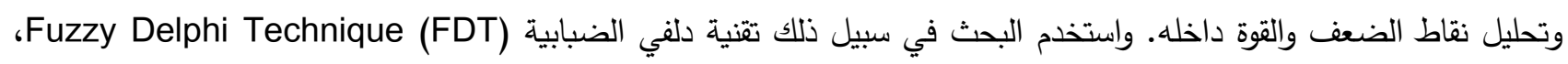
للتعامل مع مشاكل التقييمات اللفظية وتداخل المعايير وتعقيداتها وتأكيد معلومات بناء النموذج. وتكونت المعايير الرئيسية للنموذج

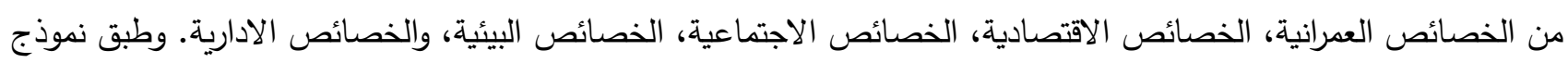
دلفي الضبابي لتقييم المجتمعات المسورة Fuzzy Delphi Evaluation Model (FDEM)، على حي الرحاب بالقاهرة كدراسة حالة، واظهر التقييم نجاح متوسط للحي في تلبية متطلبات القاطنين، وتحقيقة لاحساس الامن والسلامة بنسبة منخفضة ، وتحقيقة

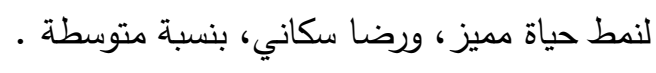

الكلمات الدالة: - 20 - n

$$
\text { المجتمعات المسورة - ندوذج تقييم - المنطق الضبابي - طريقة دلفي - مصر. }
$$

المقترحة من بوتثانان Buchanan theory

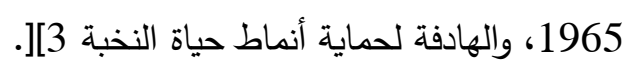

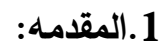

ويتكون المجتمع المسور من مجموعة مساكن ومرافق

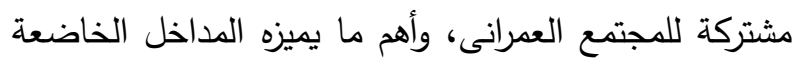
للمراقبة، كما تمثل تلك المجتمعات فرصة تسويقية للمطورين لاستثمار اثارها الإيجابية، من وجهة نظرهم، لقدرتها على

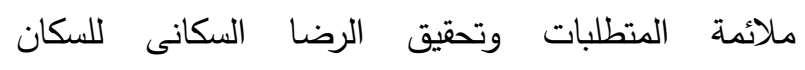

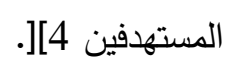

وقد ساعد في ظهور هذا النمط من المجتمعات داخل ، أن مصر، انسحاب الدولة عن تقديم الخدمات بصورة مقبولة، ابتداءا من أواخر الستينيات من القرن الماضي، أصبح خوف الساكنين من الجريمة مشكلة اجتماعية كبرى

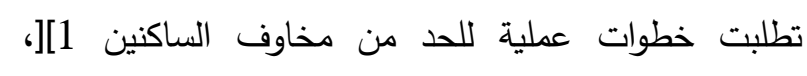
فظهرت المجتمعات المسورة كصورة للتجمعات السكنية المعبرة عن التغيرات الاجتماعية والاقتصادية بالدول، كأحد الحدات

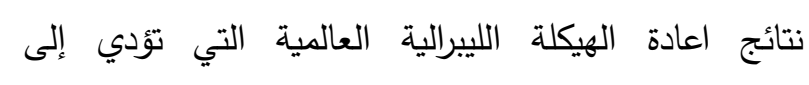
الاستقطاب الطبقي والمخاوف بثأن السلامة والأمن 2]ــــ والمجتمعات المسورة مثال للعمل الجماعي من أجل زيادة المنافع الفردية للنخب المجتمعية وتحقيق نظرية النادي

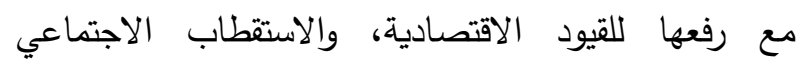




\section{Vol. 40, No. 1. January 2021}

في المجتمعات المسورة وأسباب قرارهم للانتقال لها. وتم تطبيق النموذج على حي الرحاب، كونه يصنف كأفضل

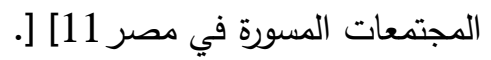

وبسبب عدم قدرة التحليل الكمي على التعامل مع التعقيدات اللغوية المستخدمة للتعبير عن الرضا، وتعدد وتداخل المتغيرات والمؤثرات في تقييم الرضا السكاني، فقد اعتمد البحث على الجمع بين تقنية المنطق الضبابي Fuzzy logic Delphi معومات غير دقيقة وغير مؤكدة، مع طريقة دلفي من فئ

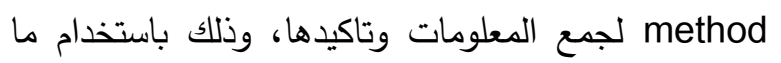

Fuzzy Delphi Method سمي باسلوب دلفي الضبابي (FDM)، الذي يستخدم لتحقيق الترابط والدمج بين المشكلة واراء الخبراء 13][. ويستخدم المنطق الضبابي لعلاج مشكلة الضبابية والعشوائية في المعلومات والتي لا تستطيع نظريات الاحتمالات التعامل معها، عندما لا يمكن تمثيل المشكلة

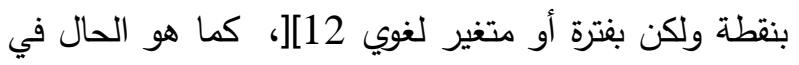
تقييم الرضا السكني. ويمثل المنطق الضبابي طريقة سهلة لتمثيل الخبرة البشرية اللفظية (كيفي) وتحويلها لقيم رياضية (كمي) يمكن تحليلها ودراستها 14][، ويزيد من كفاءة هذه الطريقة دعمها باسلوب دلفي لتحسين كفاءة النتائج وتأكيد المعلومات 15][].

\section{2. - 2. منهجية البحث :}

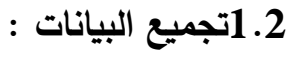

تطبق هذه الدراسة منهجية دراسة الحالة من أجل تقييم وقياس نجاح المجتمع المسور في تلبية متطلبات الرضا السكاني للقاطنين. وطبقا لدراسة لزاينال Zainal منهج دراسة الحالة يسمح للباحثين باختبار البيانات لسياق

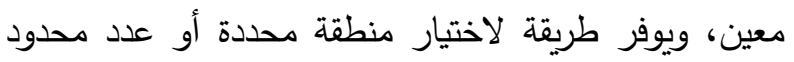
من الافراد كموضوع للدراسة، واشار البحث لدراسة اجريت في منطقة معينة وعلى مجموعة مختارة من المستجيبين،
المصاحب له، وتوجه الاثرياء للفصل الذاتي في السكن بمجتعات تحقق الحواجز المادية 5][، إضافة لعدم قدرة الحكومة المحلية ضمان الجودة الثاملة داخل المجتمعات العمرانية، كما تتامى الطلب على المجتمعات المسورة بعد ثورة 25يناير 2011 فى مصر ، والتى لجأ إليها الأفراد بحثا لماتيا عن الأمان، والخصوصية، ونمط الحياه الفاخر 6] [. وتتأثر قرارات السكان لاختيار المجتمع المسور ، بمدى هن كفاءة تلبية هذا المجتمع لمتطلبات المستخدمين وتحقيق شعورهم بالرضا 7][]، وهو ما انعكس في عدم نجاح بعض

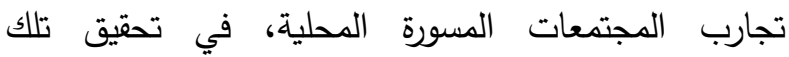
المتطلبات وانعكاسها السلبي على المجتمع السكني 8][. وتمت مناقثة رضا المستخدمين داخل المجتمعات المسورة في العديد من الدراسات السابقة التي تدرس الخصائص الشخصية، أو المميزات المادية والاجتماعية للبيئات السكنية وانعكاسها على مشاعر الرضا والسعادة، ونوعية الحياة العامة، وتلبية المسكن لمتطلبات القاطنين وخاصة الهيبة الاجتماعية prestige الدراسات عدم الرضا عن السلامة والأمن بشكل خاص.

وتتاولت دراسة لعباس واخرين 7] [ مفهوم الرضا السكنى بأنه مدى الإستجابه العاطفية للسكان لبيئتهم السكنية وما تحتويه من مكونات مادية مثل المسكن والمرافق لإنق

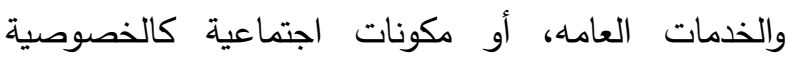
والإنتماء، وتختلف مدى الإستجابة بإختلاف التركيبة السكانية والخبرات المعيشية للسكان. وقد أشار الخبراء بأن أهم مؤشر لقياس نجاح البيئة العمرانية، هو مؤشر الرضا

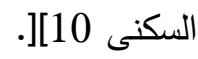

لذلك كان هدف البحث اقتراح نموذج لتقييم مدى نجاح المجتمعات المسورة داخل مصر في تحقيق منطلبات المستخدمين وتلبية توقعاتهم لتلك المجتمعات. وتم ذلك من خلال دراسة وتحليل متطلبات الرضا السكاني للمستخدمين 
Vol. 40, No. 1. January 2021

مدي تحقق المعيار Presence index(PI) في منطقة

في مرحلة لاحقة تم عرض التصميم النهائي للاستبيان الثاني على مجموعة الخبراء للتأكد من وضوح الفهم لاسلوب واسئلة الاستبيان، وتم بناءا عليه اجراء بعض التعديلات الطفيفة على التصميم والاسئلة. وتم طرح الاستبيان الثاني في صورته النهائية على العينة المستهدفة، ايضا باستخدام اسلوب (وجها لوجه) للتحفيز وتوضيح الاسئلة والحصول

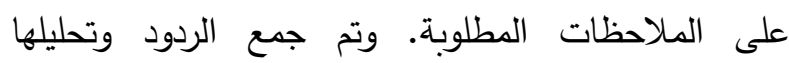
باستخدام تقنية المنطق الضبابي fuzzy logic.

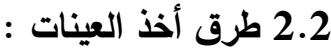

تم اختيار العينة المستهدفة للدراسة باستخدام اسلوب

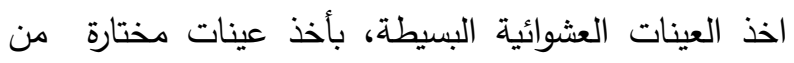
السكان بشكل عشوائي لكنه يحقق الشرائح المستهدفة لتحقيق اهداف البحث. حيث يجب اختيار المجموعات بناءا على خصائص معينة لتمثيل كامل السكان قيد الدراسة 19][ ـ في دراسة الحالة يوجد ثلاث انواع من المساكن : مساكن منفصلة (فيلات مستقلة) ، مساكن شبه منفصلة ( فيلات متلاصقة)، ومساكن مختلطة (عمارات سكنية). تم الاستعانة ببرنامج SPSS لاختيار عدد العينة باستخدام دعادلة يامنز 400 Yaman's formula شخص تمثل بشكل مقبول الانماط الثلاث للمسكن داخل

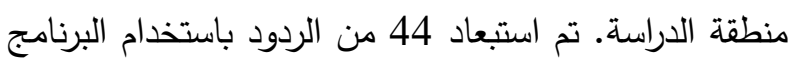
الاحصائي تمثل قيم شاذة. في الخطوة التالية تم استخدام التقنية المقترحة Fuzzy logic للحصول على النتائج المطلوبة وتم تحليل النتائج ومقارنتها بالدراسات السابقة.

\section{2 اختبار صلاحية النموذج المقترح وأهم المحدات:}

تم التحقق من صلاحية النموذج المقترح والتأكد من دقة النتائج بطريقتين، الاولى تعتمد على المنهجية المنطقية

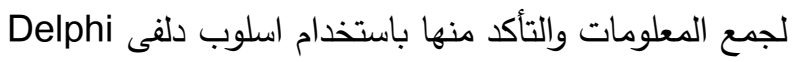

كوسيلة لجمع وفحص البيانات والمعلومات اللازمة لتحقيق

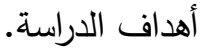

ونتيجة لنقص المعلومات المتوفرة عن المجتمعات المسورة في مصر ومتطلبات الرضا السكاني فيها، فقد تم استخدام منهجية الاستبيانات جنبا الى جنب مع الدراسة

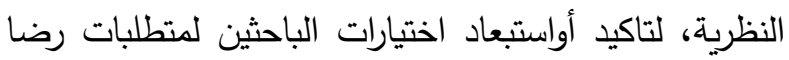
المستخدمين، داخل المجتمعات المسورة ولاختيار عناصر محددة كركائز لنجاح أي مجتمع مسور • حيث ان منهجية الاستبيان معترف بها على نطاق واسع لانتقاء المعلومات المؤكدة في بناء المعرفة 17][، واستخدم اسلوب وجها لوجه في الاستبيانات لتحفيز المجيبين وضمان (face to face) دقة الاجابات وتحسين معدل الاستجابة 18][.

واعتمدت منهجية البحث على اعداد استبيانين، الأول لتأكيد واستبعاد عناصر التقييم، واستخدم اسلوب دلفي لتقريب الاختيارات وتاكيدها، وتم طرحه على مجموعة محددة من الخبراء والمستخدمين ذوي الاقامة الممتدة لتقليل عدد دورات الوصول للقرار. أما الاستبيان الثاني لتقييم نجاح دراسة الحالة فتم باستخدام نتائج الاستبيان الأول، وبتصميم يتتاسب مع تقنية المنطق الضبابي، واشتمل على متطلبات الرضا السكاني بالمجتمعات المسورة في صفوف مقسمة الى خمس معايير رئيسية هي الخصائص العمرانية، الخصائص الاجتماعية، الخصائص الاقتصادية، الخصائص البيئية، والخصائص الادارية، وتحوي اجمالا 24 معيار فرعي. بينما تم وضع الركائز الاساسية للتقييم في أعمدة، والتي أجمعت الدراسة النظرية انها الحافز الأساسي لقرار المستخدمين بالانتقال للمجتمعات المسورة، وبالتالي نجاح هذه المجتمعات في تحقيق الرضا السكني. وتمثلت هذه الركائز في: مؤشر

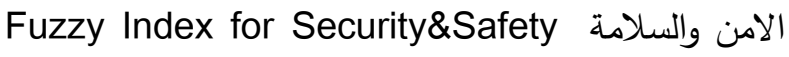
Fuzzy index for مؤشر نمط الحياة)، (FISS) Fuzzy Index Lifestyle (FIL) for Satisfaction (FIS) 
ولا يزال هاك جدل حول الآثار الاجتماعية للمجتمعات المسورة، فالمطورون يدعون انهم يقدمون ما يحتاجة المستخدمون، ويعززون شعورهم بالانتماء، بينما علماء الإجتماع يجدون أنه يفاقم ظاهرة الإستبعاد والفصل باهله الإجتماعي، ويرى المستخدمون أن تلك المجتمعات تمكنهم من الثعور بالراحة في حياتهم الإجتماعية 24] [ ـ

وقسمت دراسة لشاوش 25 Shawish]، أنماط المجتمعات المسورة الى ثلاثة: وهى مجتمع نمط الحياه Lifestyle Community

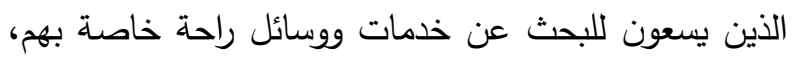
والسعى وراء بيئة اجتماعية متجانسة، مجتمع النخبة Prestige Community، وهو مجتمع يجذب السكان الذين يبحثون عن حى مستقر من حيث حماية قيمة

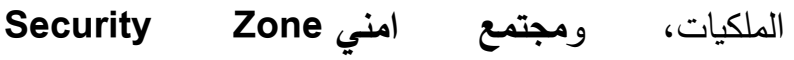
Community الذين يهددون الأمان، لانهم يفضلون الحماية.

ويرى كاو 26 Cao [ أن سبب نشوء المجتمعات

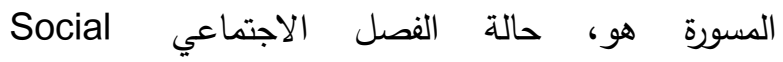
segregation

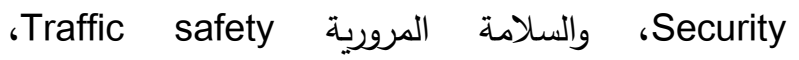
وخصخصة الاسكان Housing privatization، والخدمات

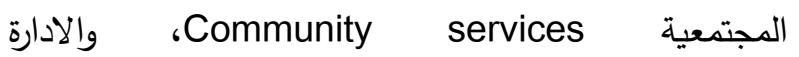
Social Management ونظام التحكم الاجتماعي، ونسي .control

وهناك عدة عوامل تؤثر على قرار المستخدمين للانتقال

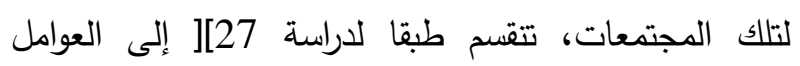

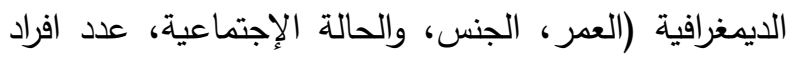

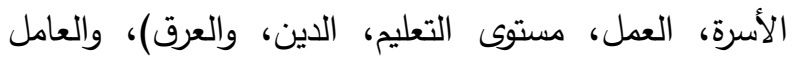
الاجتماعى الاقتصادى (دخل الأسرة واسلوب حياته)، والعامل الثقافى، وعوامل أخرى كالموقع، والمسكن، والحى، method، والثقة المثبتة بحثيا وتجريبيا في تقنية المنطق الضبابي Fuzzy logic المستخدمة للحصول على النتائج. والطريقة الثانية: بعرض النتائج على مجموعة الخبراء ومقارنة النتائج المماثلة، واقرارهم بمنطقية تلك النتائج وانها معبرة عن المجتمع المسور المستهدف، كذلك عرض النتائج على بعض القاطنين، واقروا في مناقثاتهم منطقية النتائج بشكل كبير • واقتصر البحث في بناء نموذج تقييم المجتمعات المسورة، على تقييم مدى تحقق متطلبات الرضا السكني للمستخدمين، دون التطرق للتقييم العمراني الثامل للمجتمع المسور أو تقييم إيجابيات وسلبيات تلك المجتمعات على دلى

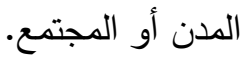
3.الاراسة النظرية والتقنيات المستخدمة:

\section{3 المجتمعات المسورة Gated Communities:}

تسمى المجتمعات المحاطة بأسوار ولها أنظمة

Gated ومداخل خاصة بالمجتمعات المغلقة أو المسورة لسوات Communities المدينة، الذى يحيط به حاجز يفصله عنه، ويقتصر الدخول على السكان والزائرين، فيكون الاخول فيها مقيدا على لهى العامه، مع توفير المساحات الخاصة بالوحدات السكنية والمساحات العامه التى يمكن مشاركتها مع السكان 20][. ويمكن تعريف المجتمعات المسورة على انها (المجتمعات ذات الابواب والحراسة لتحقيق الأمن والراحة والتجانس الاجتماعي ونوعية حياة افضل) 9][. وعموما، فان

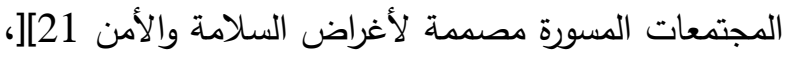
والمستخدمون مستعدون لدفع المزيد للعيث في المجتمعات

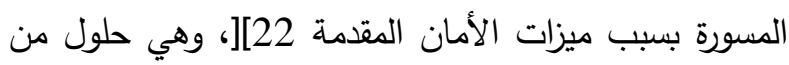
وجهة نظرهم لمخاطر الجريمة والتتهور الثقافي والمادي ونقص البنية التحتية والمساحات الخضراء بالمجتمعات التقليدية23] [، بينما يعتبر كثير من العلماء، ظهورها

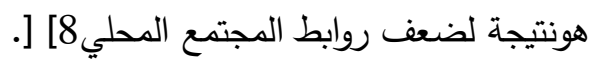




\section{Vol. 40, No. 1. January 2021}

السكانية. بينما ذكرت دراسة لعبدالله واخرين 30][ أن الانتقال يكون بغرض الحياة العصرية والعيش الآمن من خلال الاسوار التي تحميهم وتقصلهم عن الطبقات الأخرى. وترى لئري دراسة تانلكو [23 Tanulku أن الانتقال يكون بسبب ولن البحث عن نمط حياة يحقق التجانس والتميز والاستمتاع بالالعاب الرياضية ومواكبة الحداثة.

وذكرت بعض الدراسات أن العوامل التى تؤثر على تفضيلات المستخدمين داخل تلك المجتمعات المسورة، هى الخصائص الإجتماعية والإقتصادية، والجودة البيئية، والامن والسلامة، وقيمة المجتمع، والتفرد، وتوفيرالخصوصية والمناظر الطبيعية والمرافق المختلفة 9][، وعامل الاداراة والصيانة 30][، وتوفر طبقة مشتركة ثقافيا أو مهنيا وأحيانا دينيا، وانفصال المجتمع المسور من خلال عناصر مادية (الجدران والأسوار) والحواجز الثقافية ( الخلفية التعليمية والثقافية والاجتماعية) 23][. واظهرت دراسة شامسودين واخرين . Shamsudin el] بالمجتمعات المسورة كانت بوضوح للامن ثم الخصوصية ثم الموقع على الترتيب.

وتعزز هذه الحواجز الادارة الداخلية التي توفر آليات

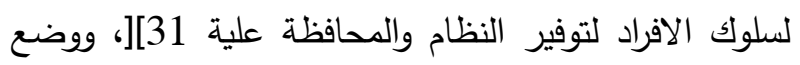
إدارات التطوير والتتمية قيود قانونية على سلوك السكان واستخدام ممتلكاتهم 32][، وتحقيق السلامة والامن وتحسين نمط الحياة والحصول على مساكن افضل ومجتمع اقل كثافة، وخدمات افضل كالمدارس والمستثفيات والملاعب

28[33,28، والحفاظ على مجتمع متماسك اجتماعيا 32][.

وحددت دراسة لتشابمان 34 Chapman] متطلبات

الرضا السكانى داخل المجتمعات المسورة، في احتياجهم للإنفصال عن باقى المدينة، التجانس الإجتماعى، المساحات الخضراء، الضوضاء، القرب من الطرق الرئيسية، البعد عن مصادر التلوث والمصانع، حصانتها ضد الجريمة، الخدمات
وتؤثر هذه العوامل على السكان بنسب متفاوته، وهناك أيضا عامل الإعلانات والتسويق التى توجه رغبة الأفراد وقراراتهم. وفى دراسة لجوزي 28 Guzey] حددت أسباب انتقال وتفضيل السكان وأسباب نمو المجتمعات المسورة فى لهى

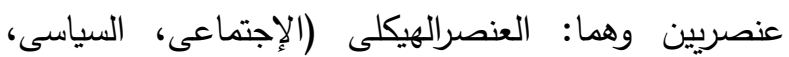
الإقتصادى) ويشمل انعدام الأمان، الخوف من الجريمة، قلة الخدمات الأساسية المتوفرة للسكان من قبل الدولة، عدم الإمان الدمان المساواة الإجتماعية، الإستقطاب الإجتماعى، والإتجاه

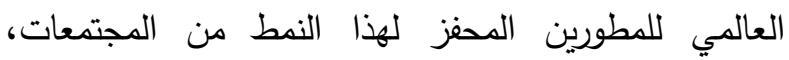
العنصر الثخصى (الدوافع، والرغبات الإجتماعية)، تحقيق نمط حياه أفضل، تجنب مشاكل المدينة، البحث عن التجانس الإجتماعى، والوضع الإجتماعى والتجديد.

Shamsudin واستتتجت دراسة لشامسودين واخرين el.

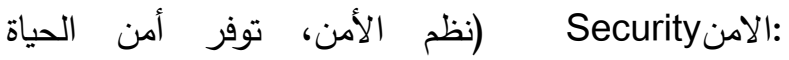
والممتلكات، الجريمة)، الخصوصية Privacy (العزل عن Facilities and الجمهورالعام، الاسوار)، المرافق والخدمات Ammonites والمراكز الرياضية، المشاركة في المرافق)، نمط الحياة الفاخر Luxurious lifestyle (حالة الثراء والتعليم الجيد، الدخل المرتفع، الاستعداد للاستمرار بنفس المستوى الطبقي)، الموقع Location (القرب من المدينة والععل والمدراس)، احساس النخبة (النفوذ) Prestige (الطبقة الاجتماعية، الغنى والثهرة)، والخدمات الأمنية المتوفرة (كاميرات المراقبة

$$
\text { والبوابات الالكترونية والحراسات). }
$$

وتحدد دراسة لموهد واخرين . Mohd el. تزايد الاقبال على تلك الانماط من المجتمعات، في انعدام الامن، الخوف من الجريمة، فثل الدولة في تلبية الخدمات الاساسية، زيادة عدم المساواة الاجتماعية، الاستقطاب الاجتماعي، وتشجيع المطورين العقاريين، كما أظهرت دور التسويق والعوامل الخارجية فى إعادة تثكيل القرارات 
التسعينات الى منطقة الساحل الثمالي غرب الاسكندرية وتحولت تدريجيا للسكان الاكثر ثراءا مع بداية الالفية الثانية. وانتقلت المجتمعات المسورة إلى القاهرة للحصول على ما لماءعاه

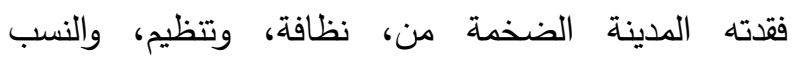
الانسانية، والمساحات الخضراء، وبحثا عن الخصوصية لهنها والفخامة والرفاهية 40][، وأظهرت دراسة لكسيبة 8] [ عدم تلبية بعض من المجتمعات المسورة لكثير من متطلبات المستخدمين سواء في التصميم الداخلي للنماذج المعمارية،

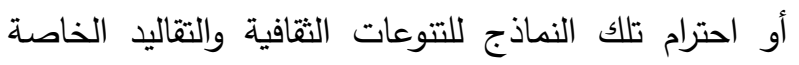
بالمستخدمين، واحيانا وجود تفاصيل ومفردات معمارية مبالغ فيها لا ترضي الاذواق العامة.

وتختلف المجتمعات المسورة المصرية عن تلك

الموجودة في اسطنبول وطهران وبيروت من حيث انها لا تزال تستهدف بالاساس السوق المحلية، وأنها أشبه بمثيلتها الأمريكية والأوروبية كونها بنيت حول المناطق الترفيهية وملاعب الجولف 38][. وتحقق تلك المجتمعات المصرية للمستخدمين، الراحة، التميز الاجتماعي، الوضع المرموق، ولها،

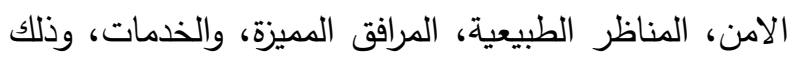
على النقيض من مدينة القاهرة التي أصبحت مزعجة ولا

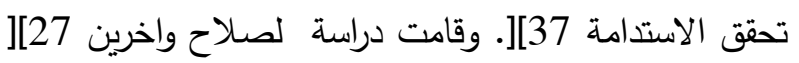
بعمل مقابلات شخصية مع عدد من سكان المجتمعات المغلقة فى مدينة الإسكندرية، والسؤال عن نمط حياتهم والميزات التى توفرها لهم المجتمعات المسورة، ومن خلال المقابلات تم استتتاج بعض العوامل التى تؤثر على تلى الافضلية عند السكان، وهى المناطق الخضراء والترفيهية (Landscape and recreational Lifestyle المناطق المفتوحة Open spaces، الثعور بالأمان Feeling safe، وجود البوابات Gates، التجانس الإجتماعى Similarity withe neighbors، لبالكمان الكثافة Density الإستعمالات المختلطة Mixed uses، وعلاقات الجوار Friendship with neighbors.
العامه، التجانس الإجتماعى، مرافق البنية الأساسية، مدة الإقامة، الحيازة (التملك)، والميزات الإقتصادية. وفي دراسة لمسيون 35 Misun] تمت دراسة عناصر تحقيق الرضا السكانى بالمجتمعات المسورة وتتاولت عوامل مثل، العوامل الديموغرافية، كثافة السكان، التواصل، مدة البقاء، النشاط

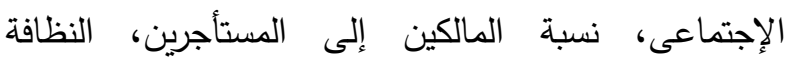
والمناطق الخضراء، وصول المشاه إلى الخدمات التجارية، حركة المرور، المسافة إلى العمل، المسافة إلى الأصدقاء

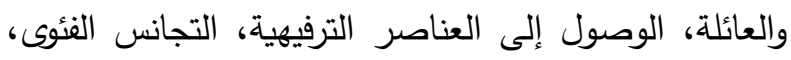

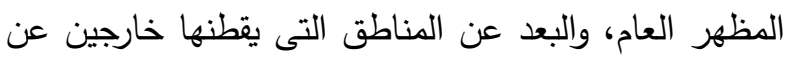

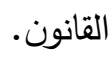

\section{3 المجتمعات المسورة في مصر :}

انتشرت المجتمعات المسورة في الدول العربية ومصر على وجه الخصوص، كنوع من الوجاهة الاجتماعية والتميز الذي يحقق الخصوصية 30][. ومع توسع القاهرة الكبرى ظهرت العديد من المجتمعات المسورة في الصحراء شرقا وغربا، كنوع من حلم الترف الاجتماعي، الذي تسعى له

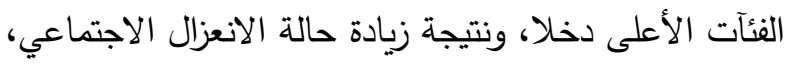
وهربا من إهمال وتدهور وسط المدينة 8][، شكل (1). وقد بلغت اكثر من مائة مجتمع مسور على اطراف القاهرة الكبرى 24][ نتيجة لبيع الدولة مساحات شاسعة من الأراضي المملوكة للدولة إلى المستثمين 36] [ب، بالاضافة لارتفاع النمو الاقتصادي الناتج عن التحرر الاقتصادي لادي وفرض سياسة الخصخصة 37][، ونتيجة استعداد القطاع العقاري لاستيعاب السيولة الزائدة للمصرين الذين يفضلون الاستثمار في العقارات معتبرا أنها منخفضة المخاطر مقارنة

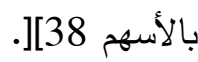

وفكرة المجتمعات المسورة ليست جديدة على مصر، فقد ظهرت في القاهرة الفاطمية، التي كانت ككل مدينة مسورة 39][، ثم انتقلت للظهور في الثمانينات في مدينة الاسكندرية كمنتجعات ترفيهية بدأت بالمعمورة، ثم انتقلت في 
Vol. 40, No. 1. January 2021

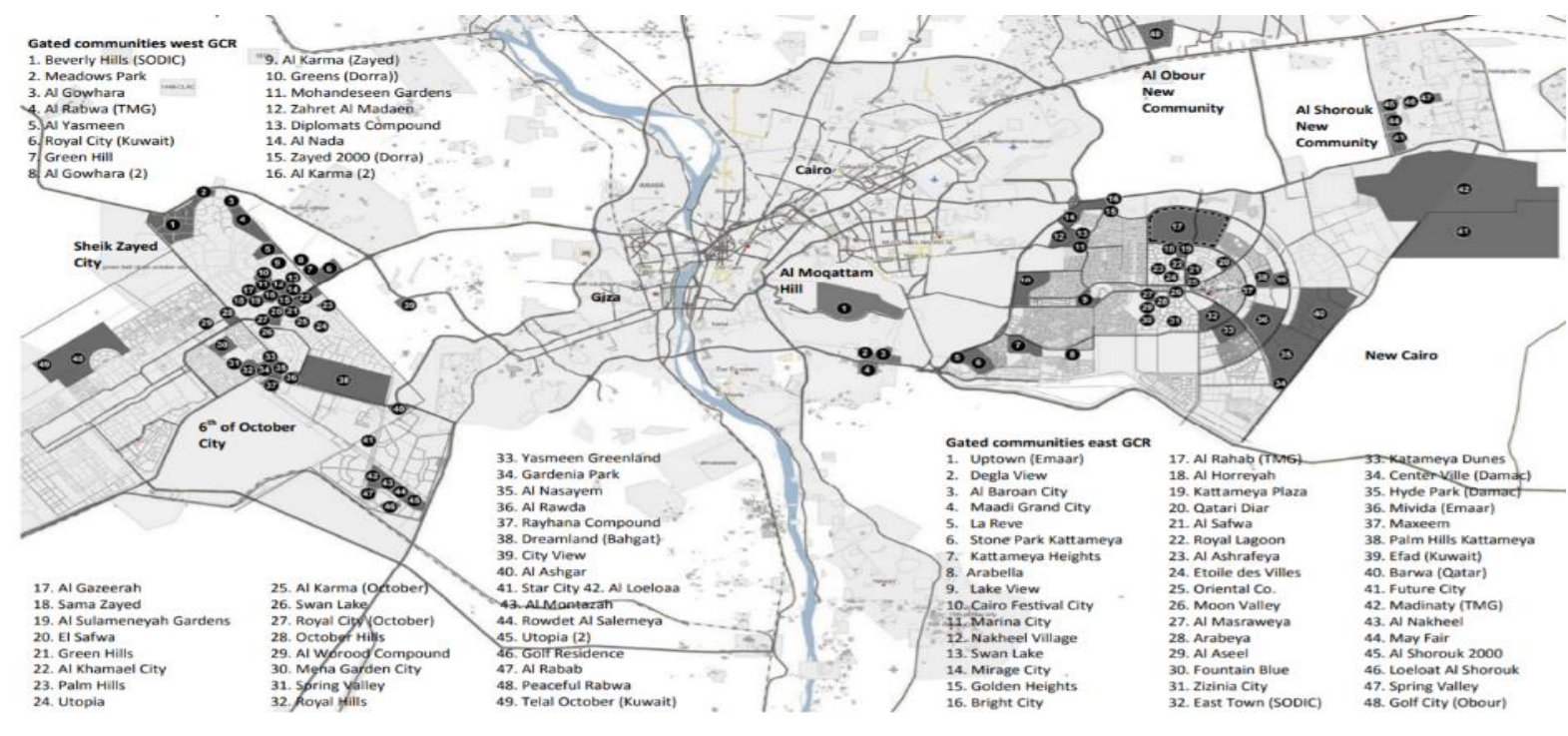

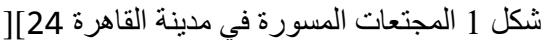

مستوى الاستجابه، وخفض عدد جولات الاستبيان، والتعامل

مع ضبابية معلومات المجيبين 15][.
Fuzzy Delphi 3.3

:Method (FDM)
ان اللجوء الى طريقة المنطق الضبابي Fuzzy يحل مشكلة الغوض وعدم اليقين وعدم الدقة في اراء الخبراء الثخصية، ويحل المشاكل التي ليس لها قيم محددة 12][، وذلك عن طريق التعامل مع المنطقة الرمادية غير الواضحة لاجابات المستجيبين التي تتميز بها الاسئلة عن الرضا والحزن والفرح 14][. ففي ضوء عدم وجود نموذج رياضي يمكنه ان يصف سلوك نظام، تصبح طريقة

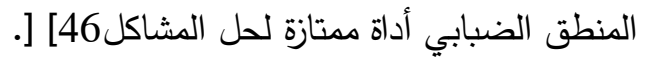

ويعتمد المنطق الضابي على تحديد المتغيرات اللغوية، وشكل الدالة العضوية المستخدمة، والقواعد المنطقية ( إذا- إذن) لتوضيح العملية الضبابية بطريقة تماثل سلوك النظام المستهدف، ويعتمد النظام على ثلاث مراحل اساسية هي: التضبيب Fuzzification، ومولد الاستدلال Fuzzy

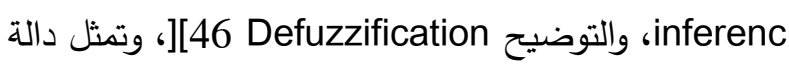
العضوية في المنطق الضبابي، حالة عدم اليقين، وتكون في المدى [0،10] بانتقال تدريجي. وتتشأ دالة العضوية لاعطاء معنى عددي لكل مقياس لفظي (منخفض جدا، منخفض،
طريقة دلفي التقليدية، هي عملية هيكلية تستخدم مجموعة من الجولات لجمع المعلومات وتأكيدها، وتستمر هليهريه group العملية حتى يحدث اجماع من مجموعة الخبراء consensus دلفي طريقة بحث نوعية موثوق بها مع امكانية حل

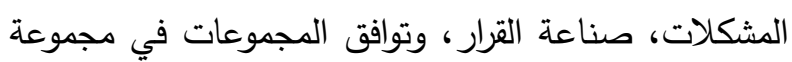
واسعة من المجالات 42][، وعلى الرغم من تلك المزايا فيعيبها مشكلة الغموض وعدم اليقين في اسئلة المسح الميداني والاجابات 43][، كذلك انخفاض اتساق أراء الخبراء، وارتفاع كلفة تعديل تلك الاراء لتحقيق اراء جماعية متسقة. لذا اقترح موراي Murray تطبيق طريقة دلفي الضبابية Fuzzy Delphi method للقضاء على الغموض لطوريق .][44 ويساعد الجمع بين اسلوب دلفي واسلوب المنطق الضبابي، في الوصول الى التقارب المطلوب والجمع بين وجهات نظر المستجيبين في الموضوعات الضبابية غير يقينية الاجابة [45] ، وتجنب تشويه رأي الخبراء، وتحسين 
Impact Index for في زيادة الرضا السكاني Increasing Satisfaction (IIS) بين مؤشر مدى التحقق (PI))، ومؤشر الامن والسلامة

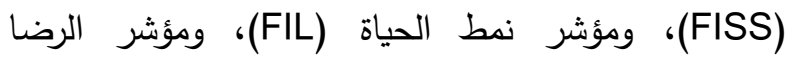
السكاني (FIS)، لاعطاء مؤشر دلفي الضبابي لتقييم التجمع

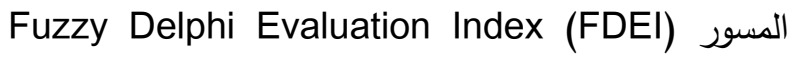

$$
\text { وهو المؤشر الرئيسي للبحث. }
$$$$
\text { 5. دراسة الحالة ( حي الرحاب) : }
$$

بدأ إنشاء الرحاب في سنة 1997م كأحد أبرز

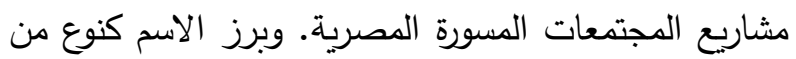
التسويق العقاري باستخدام كلمة عربية تعبر عن الرحابة والكرم، كوسيلة لجذب طبقات النخبة، حيث تم تخطيطها في لعيد البداية لاستيعاب 120 الف نسمة، وتم زيادة المستهدف بـ 80 الف نسمة أخرى مع عمليات التطوير في التصميمات

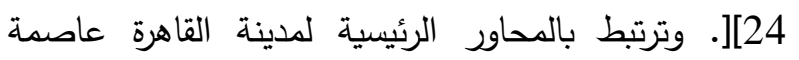

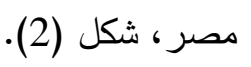

يتكون مخطط المشروع من 10 مناطق سكنية، تقدم أنماط مختلفة من الوحدات السكنية تتوعت بين الفيلات شبه

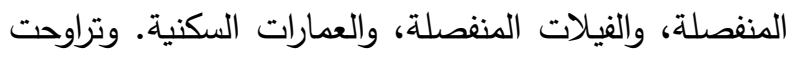
مساحات الثقق السكنية بين 60 و 320 م2، مكونة من 52 بالمساحات الخضراء، والربط بينها بشبكة متميزة من مسارات المشاة. بينما كانت النماذج الأولية للفيلات 26، تراوحت لئل مساحاتها بين 170م2 للفيلات شبه المنفصلة، وصولا لمساحة 52052 للفيلات المنفصلة الفاخرة، مع حدائق تمثل 60 من مساحة الأراضي على الأقل 55][، شكل (3)، شكل (4).
متوسط، عالي، عالي جدا) تقابلها في دالة العضوية

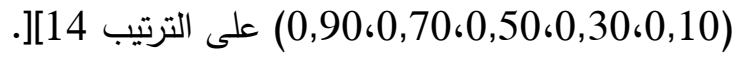
وقد استخدمت هذه الطريقة في التقييم من كثير من حثن الباحثين امثال ميرت 47] في تقييم جودة الأحياء السكنية، وزايد 48][ في تقييم الانتاجية، وتشانج 49]] في تقييم مخاطر المشاريع، وما 50] في تقييم الموزعيين التجاريين، واستخدمه هاشمي 51][ في وضع المؤشرات الحضرية لطهران، وطبقه جايسري 52][ على التخطيط الحضري، وطبقه جاديري 46][ على التتمية المستدامة للددن، وطبقه ألكان 53] [ على الواجهات الحضرية.

4. بناء نموذج دلفي الضبابي للتقييم Fuzzy :Delphi Evaluation Model (FDEM)

طبقا لمنهجية البحث فقد تم الاستقرارعلى المعايير

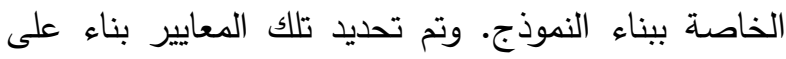
الدراسة النظرية ونتائج الاستبيان الأول طبقا لما تم ذكره بالمنهجية، جدول (1)، وقد استخدم اسلوب العصف الذهني، كأحد الأساليب المعترف بها للحصول على المعرفة وتأكيدها [54]، بعقدعدة جلسات مع الخبراء لبناء القواعد المنطقية لنظام التقنية الضبابية واجراء التعديلات اللازمة عليها للوصول لأفضل بناء لقواعد النموذج.

وتم في البحث استخدام النموذج المقترح (FDEM) باربعة طرق، الأولى الربط بين مؤشر مدى التحقق (PI)، ومؤشر الامن والسلامة (FISS)، لاعطاء مؤشر التأثير في

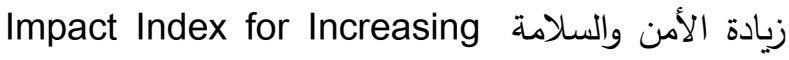
Security\&Safty (IISS) مؤشر مدى التحقق (PI)، ومؤشر نمط الحياة (FIL)،

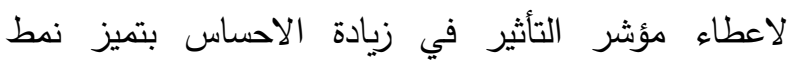
Impact Index for Lifestyle (IIL) الحياة لاعيرة Increasing الطريقة الثالثة، الربط بين مؤشر مدى التحقق (PI)، ومؤشر الرضا السكاني (FIS)، لاعطاء مؤشر التأثير 
Vol. 40, No. 1. January 2021

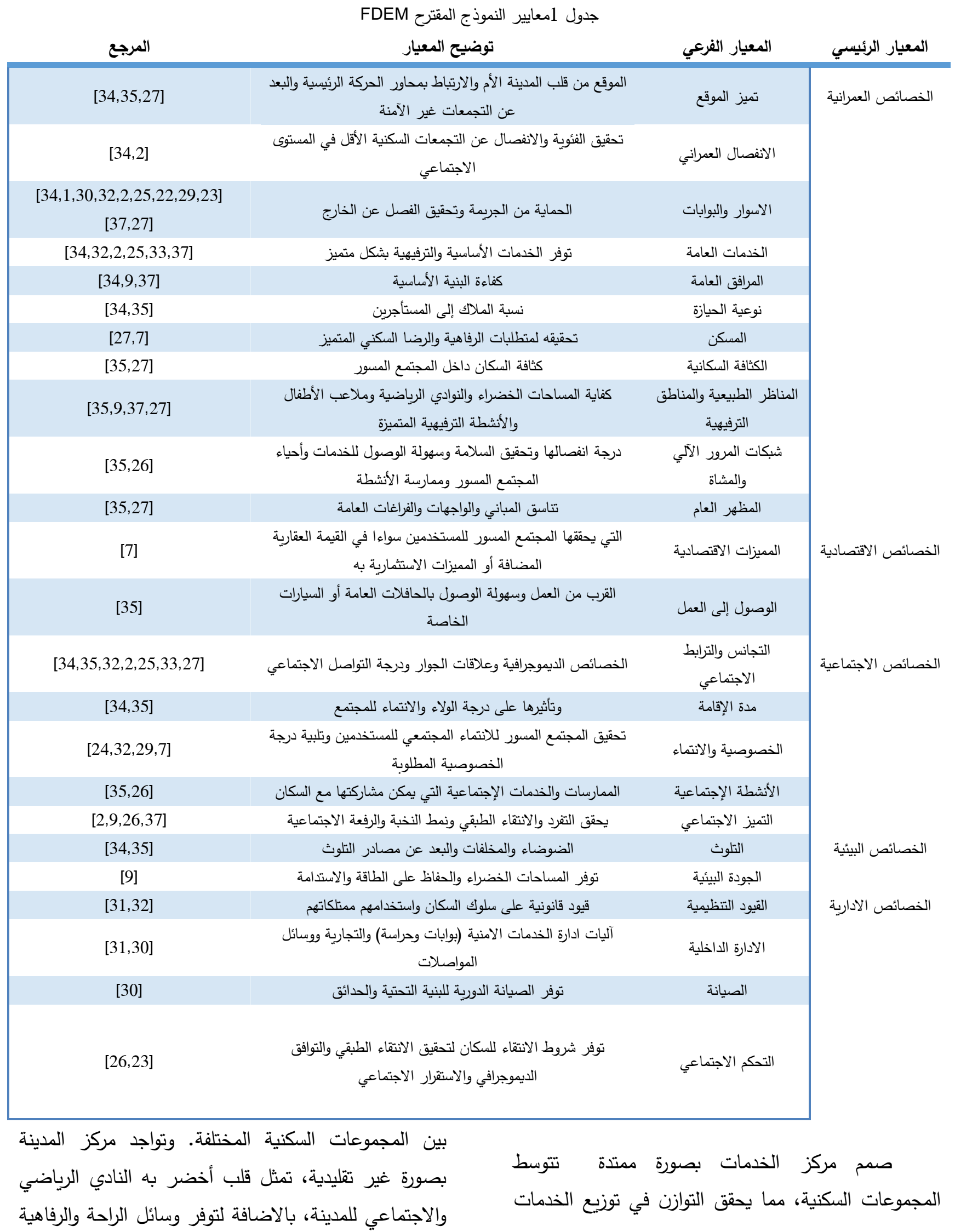


Vol. 40, No. 1. January 2021

والسباكة والاتصالات وغيرها ، وتوفير الحافلات للانتقال من وإلى حي مدينة نصر وحي مصر الجديدية بمدينة القاهرة، وتم وضع قواعد تقييدية للسلوك المشترك بين السكان بما يحقق مجتمعا هادئا خالي من المشاكل والتشوهات العمرانية، كما يدفع السكان مبالغ سنوية نظير الصيانة السنوية

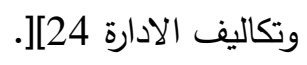

كانت خطة التسويق تعتمد على التطوير الدائم لمواكبة متطلبات ورغبات السكان واستيعاب المتغيرات المحيطة، فتم تغيير المساحات المقدمة للتسويق واعادة تصميم البعض لتناسب المتطلبات، مما ساعد في وصول معدل الاشغال للاربع مراحل الاولي الى 65\%، وهي نسبة

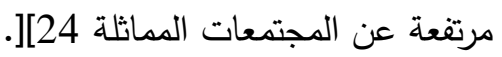

أظهرت دراسة قام بها فجال 11][، أن هنالك عدد من المشكلات التي تواجه حي الرحاب، مثل هيمنة حركة

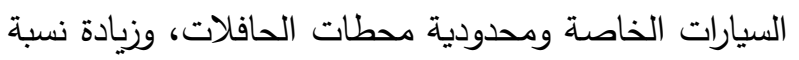
الطرق مما يؤدي لتأثير سلبي على البيئة، وعدم كفاية أشجار التظليل لتشجيع حركة المشاة والدراجات، وعدم اعتناء

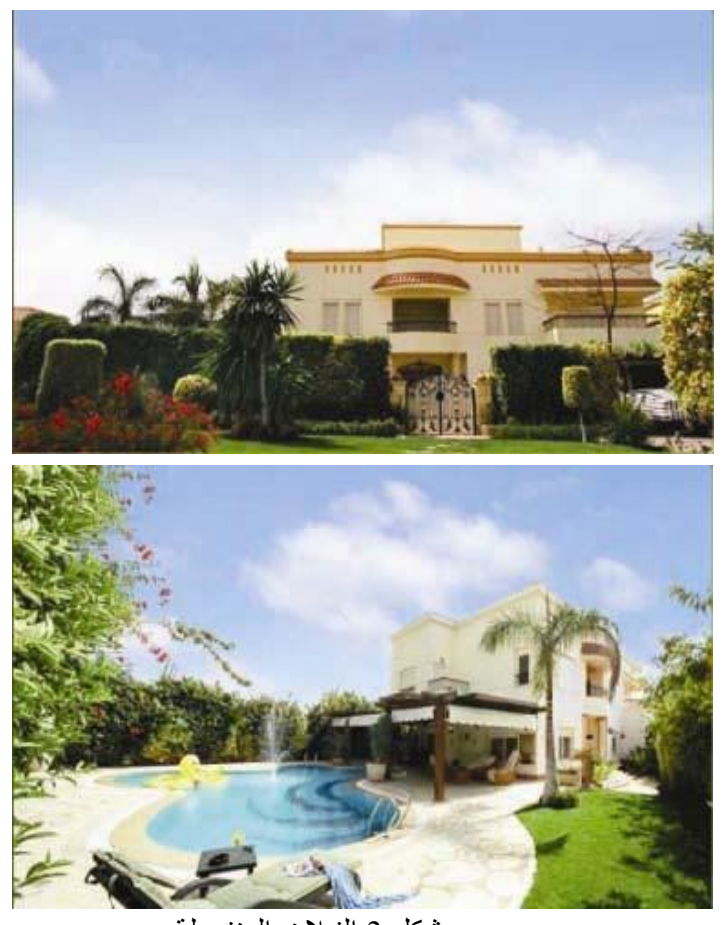

شكل 3 الفيلات المنفصلة
ومرافق الخدمات التي تشمل عددا من المدارس الدولية، والمركز الطبي ومراكز التسوق، والمطاعم المتخصصة، والخدمات الترفيهية كالنوادي ودور السينما والمحلات

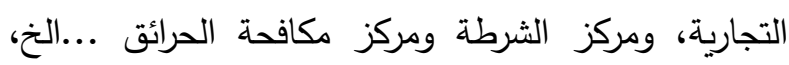
وكذلك توفرت بنية تحتية عالية الجودة 24][، شكل (5) . وضع المطورون نظاما لادارة تتسيق المساحات المفتوحة والخاصة، وجمع القمامة ومكافحة الآفات والقوارض، ونظام للامن يتوفر به بوابات وحراس منتشرون على البوابات ووسط المجموعات السكنية المختلفة، وشبكة انترنت خاصة بالسكان مع توفير فرق صيانة للكهرباء

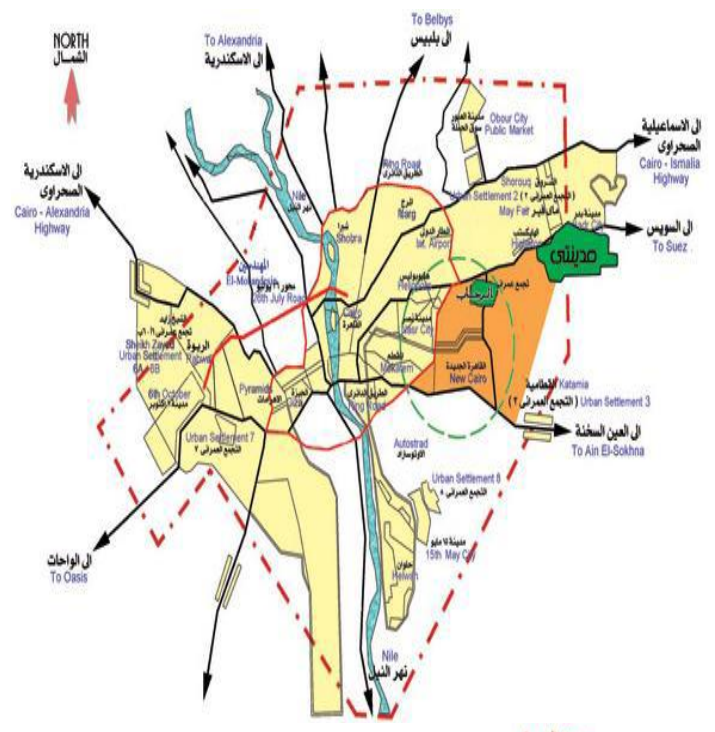

ملهن الثررول

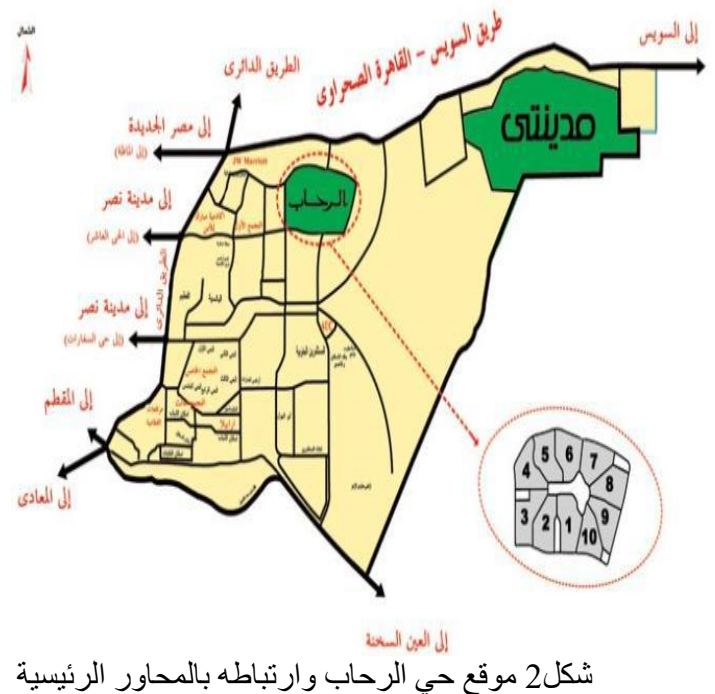


احساس متوسط بتميز نمط الحياة ، وقيموا مؤشر (IIS) بـ \%3,3، ويعني احساس متوسط بالرضا عن المجتمع

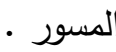

تخالف بعض هذه النتائج ما هو متعارف عليه اجمالا

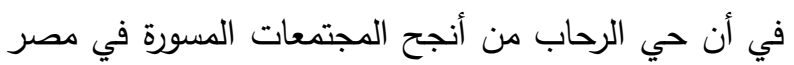
طبقا لبحث فجال 11][، لكنها أيضا ربما تشير لما أشار له له نفس البحث من وجود مشكلات داخل الحي.

وقد تمت مقابلات شخصية لمناقشة تلك النتائج مع بعض القاطنين خاصة من أصحاب الاقامة الطويلة منذ بداية نشأة الحي، وكانت أبرز أسبابهج، أن ضعف تقابن القييم الاحساس بالامن والسلامة، هو نتيجة لعدم توفر أسوار بشكل يحقق أمان كامل، بالاضافة لعدم توفر كاميرات مراقبة بالحي، والاهم هو زيادة الدخلاء على الحي بشكل كبير

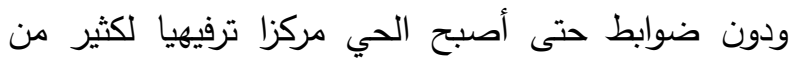

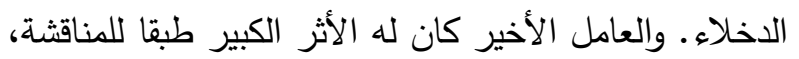
في الاحساس المتوسط بتميز نمط الحياة عما كان عليه الحال سابقا، مع ما يسببه الدخلاء من ازعاج وضوضاء. بالاضافة لارتفاع نسبة المستاجرين من انماط ثقافية مختلفة

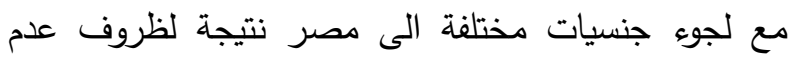
الاستقرار السياسي بدولهم، وهو ما أضاف أفراد لا تتنمي

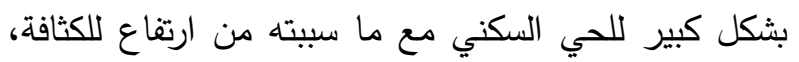
وما يتبعه من ضغط على البنية التحتية والخدمات المقدمة وجودتها، وزاد من تللك الضغوط نشوء مجتمعات جديدة محيطة بحي الرحاب أثرت في جودة خدماته وبنيته التحتية، وهو ما انعكس أيضا على حالة الرضا المتوسطة، والنجاح المتوسط للحي بشكل اجمالي.

ويفسر تلك النتائج النظر ببعض التفصيل الى النتائج

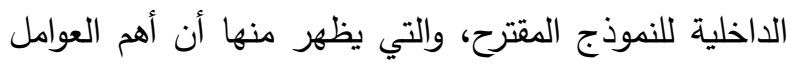
التي تحقق هذا المستوى من الاحساس بالامن في حي

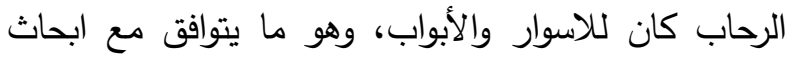

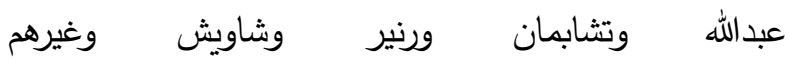
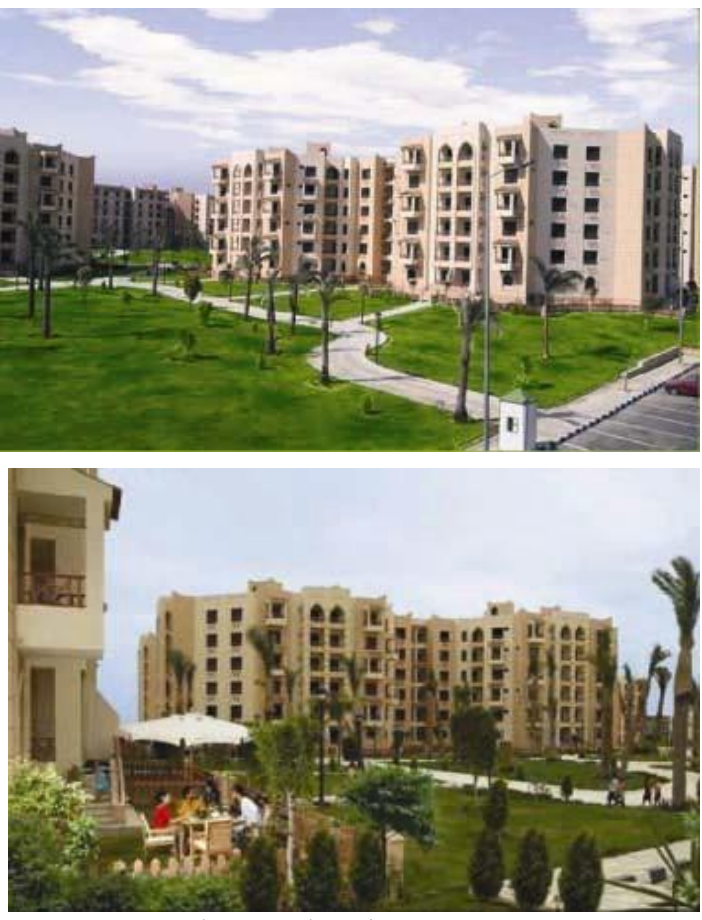

شكل 4 العمار ات السكنية

التصميم بتحقيق حلول موفرة للطاقة، كما أن عمليات التطوير أثرت على نسبة المساحات الخضراء بالحي، ولا تتوفر حلول مستدامة لاعادة استخدام وتدوير المياة، ولا نظام متكامل لجمع وتدوير النفايات، واخيرا فتصميم الحي من وجة نظر الدراسة لا يفي بمبادئ التصميم البيئي المستدام، على ولى الرغم من النظر إليه على أنه من أنجح المجتمعات المسورة لئم لئي

\section{6. النتائج والمناقشة:}

يظهر جدول (2) نتائج تطبيق النموذج المقترح على حي الرحاب. ويظهر الصف الأخير من الجدول نتيجة التثييم المستهدف للبحث، وذلك عن طريق مقارنة اجمالي نتائج المؤشرات مع القيمة المقابلة في دالة العضوية للنموذج المقترح، ويظهر منها أن عينة المستخدمين قيموا المؤشر الرئيسي (FDEI) الذي يمثل تقييم نجاح المجتمع المسور لحي الرحاب بـ 54,02\%، والذي يعني مستوى نجاح متوسط في تلبية الرضا السكني، بينما قيموا مؤشر (IISS) بـ \%38,3، والذي يعني مستوى منخفض للاحساس بالأمن والسلامة، كما قيموا مؤشر (IIL) بـ 47,3\%، والذي يعني 


\section{Vol. 40, No. 1. January 2021}

المؤشر الرئيس (FDEI)، يظهر من نتائجه أن

العوامل الاقوى في نتيجة تقييم الحي، كانت أيضا للاسوار والأبواب، ثم المناظر الطبيعية والمناطق الترفيهية، والجودة البيئية. وهي نتائج تتوافق مع الدراسة النظرية ومدى أهمية تلك العوامل في المجتمعات المسورة .
[,29,22,25,32,30,1[34 والمذكورين بالدراسة النظرية، في كونه الرغبة الاساسية لسكان المجتمعات المسورة. وياتي

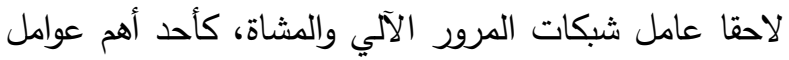
تحقيق أمن وسلامة القاطنين وهذا يوافق ابحاث الدراسة

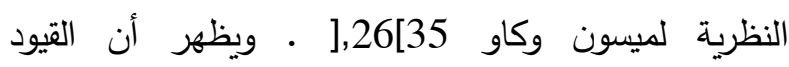
التنظيمية، والادارة الداخلية جاءا متأخرين في الترتيب على التوالي. وهو ما فسرته مناقشة بعض السكان بانه نتيجة

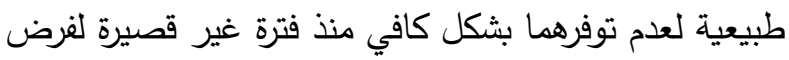
الانضباط السلوكي للسكان والزائرين وتحقيق الامان الكافي.

وعند الانتقال لفهم وتحليل نتائج مؤشر نمط الحياة،

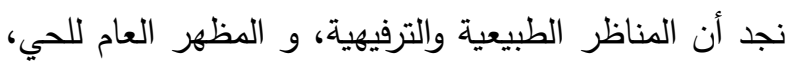
والخدمات العامة، في مقدمة العوامل التي تساهم في احساس التهاس القاطنين بتميز نمط الحياة الخاصة بهم، وهو يوافق ابحاث، تشابمان وشاويش وزهاو [3,37,27,33,25[34، بينما ياتي ترتيب عوامل كعامل وفئل

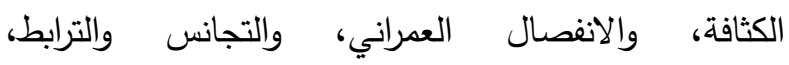

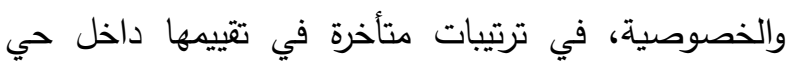

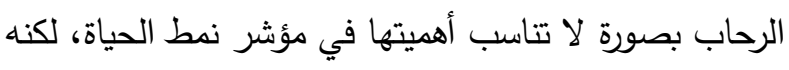
يؤكد ما ذكر سابقا في مناقشة السكان المقيمين، من مشاكل بحي الرحاب نتيجة ارتفاع نسبة المستأجرين غير المنتمين

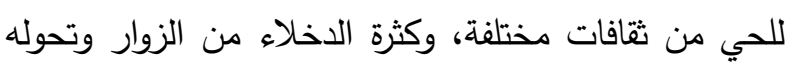
لمركز ترفيهي لكثير من سكان المجتمعات الناشئة حديثا حلول محيطه..

أما مؤشر الرضا السكاني فيظهر أنه كان أفضل حالا من سابقيه، وان عامل، الاسوار والبوابات، والمظهر العام

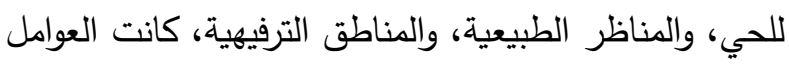

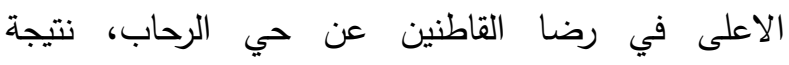

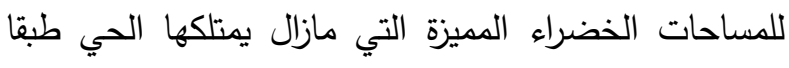

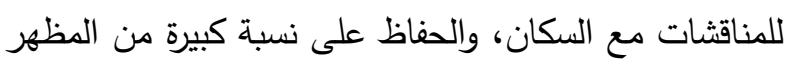

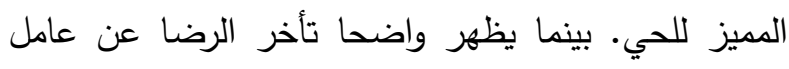

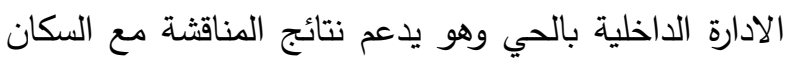
ونتيجة نفس العامل بمؤشر نمط الحياة. 
Vol. 40, No. 1. January 2021

جدول 2 نتائج نموذج دلفي الضبابي للتقييم (FDEI)

\begin{tabular}{|c|c|c|c|c|c|c|c|c|c|}
\hline الترتيب & FDEI & الترتيب & IIS & الترتيب & IIL & الترتيب & IISS & المعيار الفرعي & المعيار الرئيسي \\
\hline 5 & 54.2 & 5 & 55.2 & 11 & 41.3 & 10 & 42.1 & تميز الموقع & \multirow{11}{*}{ الخصائص العمرانية } \\
\hline 21 & 41.2 & 14 & 45.2 & 13 & 40.1 & 14 & 33.2 & الانفصال العمراني & \\
\hline 1 & 62.4 & 1 & 63.1 & 16 & 38.4 & 1 & 58.3 & الاسوار والبوابات & \\
\hline 9 & 51.2 & 7 & 53.2 & 3 & 54.2 & 16 & 29.3 & الخدمات العامة & \\
\hline 22 & 40.2 & 24 & 38.2 & 14 & 39.1 & 17 & 24.2 & المرافق العامة & \\
\hline 15 & 46.1 & 18 & 42.3 & 23 & 29.2 & 4 & 47.2 & نوعية الحيازة & \\
\hline 12 & 50.1 & 8 & 52.1 & 5 & 51.4 & 5 & 47.1 & المسكن & \\
\hline 20 & 41.3 & 17 & 43.1 & 17 & 37.4 & 18 & 24.2 & الكثافة السكانية & \\
\hline 2 & 60.1 & 3 & 59.2 & 1 & 64.1 & 15 & 33.2 & المناظر الطبيعية والمناطق الترفيهية & \\
\hline 11 & 50.7 & 12 & 46.2 & 8 & 45.1 & 2 & 51.2 & شبكات المرور الآلي والمشاة & \\
\hline 8 & 51.5 & 2 & 60.2 & 2 & 54.6 & 23 & 15.3 & المظهر العام & \\
\hline 14 & 47.2 & 16 & 44.5 & 24 & 25.1 & 21 & 19.2 & المميزات الاقتصادية & \multirow[t]{2}{*}{ الخصائص الاقتصادية } \\
\hline 23 & 36.3 & 23 & 39.3 & 18 & 36.2 & 22 & 16.3 & الوصول إلى العمل & \\
\hline 10 & 51.1 & 15 & 45.2 & 21 & 33.2 & 11 & 40.1 & التجانس والترابط الاجتماعي & \multirow[t]{5}{*}{ الخصائص الاجتماعية } \\
\hline 24 & 33.4 & 21 & 40.1 & 22 & 30.1 & 13 & 35.1 & مدة الإقامة & \\
\hline 16 & 45.1 & 20 & 40.4 & 19 & 35.2 & 12 & 37.1 & الخصوصية والانتماء & \\
\hline 18 & 44.7 & 19 & 40.6 & 20 & 34.2 & 7 & 45.1 & الأنشطة الإجتماعية & \\
\hline 3 & 59.2 & 6 & 55.1 & 6 & 50.3 & 3 & 48.2 & التميز الاجتماعي & \\
\hline 13 & 48.3 & 11 & 46.3 & 15 & 38.9 & 20 & 22.5 & 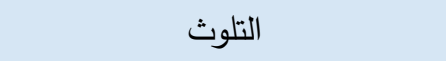 & \multirow[t]{2}{*}{ الخصائص البيئية } \\
\hline 4 & 54.6 & 4 & 56.9 & 9 & 44.5 & 24 & 13.5 & الجودة البيئية & \\
\hline 19 & 44.7 & 13 & 46.1 & 12 & 40.3 & 9 & 43.1 & القيود التنظيمية & \multirow[t]{4}{*}{ الخصائص الادارية } \\
\hline 17 & 45.1 & 22 & 40.1 & 10 & 44.3 & 6 & 45.2 & 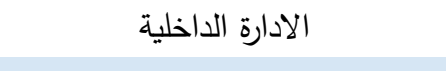 & \\
\hline 7 & 52.1 & 10 & 47 & 7 & 50.2 & 19 & 23 & الصيانة & \\
\hline 6 & 53.5 & 9 & 51.8 & 4 & 52.1 & 8 & 44.2 & التحكم الاجتماعي & \\
\hline
\end{tabular}

\section{$\begin{array}{llll}54.02 & 53.3 & 47.3 & 38.8\end{array}$}

جمالي تقييم حي الرحاب

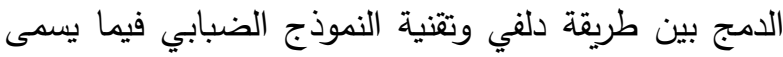

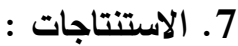

بطريقة دلفي الضبابية، وذلك من أجل التعامل مع تعقيدات وتداخل المعلومات، والتعامل مع القيم الكيفية التي تمثل مشاعر واحاسيس القاطنين نحو مجتمعهم المسور لتحويلها لقيم كمية يمكن قياسها وتحليلها. وتم بناء النموذج من خمسة معايير رئيسية: الخصائص العمرانية، الخصائص

في هذا البحث تم بناء نموذج مقترح لتقييم مدى نجاح المجتمعات المسورة في مصر في تلبية متطلبات القاطنين. وفي سبيل تحقيق بناء جيد للنموذج، تم تجميع

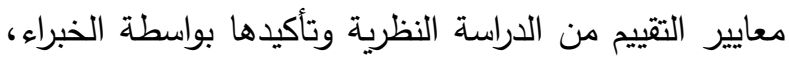
كما تم الاعتماد على أساليب احصائية حديثة عن طريق التئي 
المجتمع المسور ، والحفاظ على مستوى مرتفع لقرار السكان للانتقال اليه. وهذا يساعد في استخدام النموذج المقترح للتتبؤ لترائ بمتطلبات السكان عند انثاء تجمع مسور جديد، وينعكس في نجاحه الفعلي لتلبية متطلبات القاطنين.

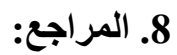

[1] Renauer, B.: Reducing Fear of Crime: Ctizen, Police or Government Responsibility? Journal Police Quarterly 41-62(1), 10(1), 41- (2007)

[2] Roitman, Sonia: Who Segregates Whom? The Analysis of a Gated Community in Mendoza Argentina,Journal of Housing Studies 20(2), 303-321 (2005)

[3] Manzi, T., Smith-Bowers, B.: Gated communities as club goods:Segregation or social cohesion? Housing Studies 20(2), 345359 (2005)

[4] Roitman, Sonia: Gated communities: Definitions, causes and consequences. Gated communities: Definitions, Urban Design and Planning 163(1), 31-38 (2010)

[5] Glasze, G, Webster, C., Frantz, K.: Private cities: Global and local perspectives. New York: Routledge. New York: Routledge (2006)

[6] Metwallly, Magda, Abdalla, Sahar: Major Trends of the Gated Communities Development in Egypt an Approach to Urban Sustainability. In : International ConferencePrivet Urbana Governance and Gated Communities, Brighton,UK (2013)

عباس, سناء, شلال, صبا: رضا الساكنين في البيئة السكنية. [7 السئة

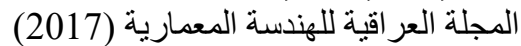

[8] Kesseiba, Karim: Cairo's Gated Communities: Dream homes or unified houses. In : Procedia - Social and Behavioral Sciences, Chung-Ang University, Seoul, S. Korea, vol. 170, p.728 738 (2015)

[9] Mohd, Thuraiya, Johari, Noraini, AbdulGhani, Rohaya: SATISFACTION LEVEL OF GATED AND GUARDED COMMUNITY
الاجتماعية، الخصائص الاقتصادية، الخصائص البيئية، والخصائص الادارية، تحوي اجمالا 24 معيار فرعي. واعتمد تقييم المعايير داخل النموذج المقترح على مدى تحقيق كل منها لثلاث ركائز أساسية هي مؤشر الأمن والسلامة،

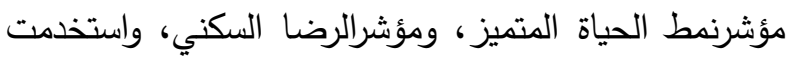
تقنية المنطق الضبابي للحصول على تأثير كل معيار في زيادة الأمن والسلامة ، والتأثير في زيادة الاحساس بتميز

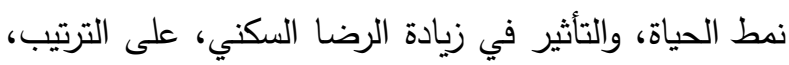
بالاضافة للحصول على مؤشر دلفي الضبابي لتقييم نجاح المجتمعات المسورة.

وفي سبيل التحقق من صحة النموذج وتقييم كفاءته، تم التطبيق على حي الرحاب بالقاهرة، وأظهر التقييم نجاح متوسط للحي في تلبية متطلبات القاطنين بصورة عامة، وتحقيقه لاحساس الامن والسلامة بنسبة منخفضة، وتحقيقه

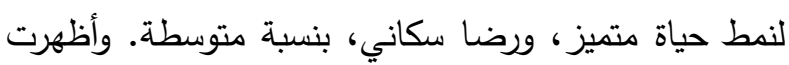

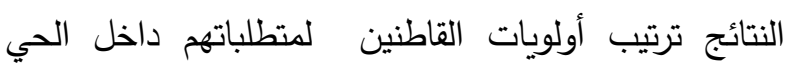
ونقاط الضعف والقوة داخله مما يساهم في تطوير الحي المستهدف، كما تساهم النتائج بصورة عامة في فهم أكبر

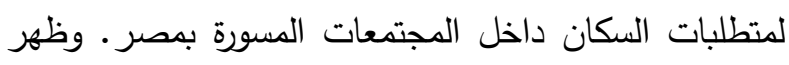
من تطبيق النموذج على حالة الدراسة، وما صاحبه من مناقشات مع السكان لفهم النتائج، أن مدة الاقامة لها جانب كبير في تقييم المجتمع، والمقارنة بين ما يتم فقده من مميزات تؤثر بشكل كبير على حالة الرضا السكني، باكثر لئر منها في حالة الاقامة الاقصر مدة. وتم التأكد من قوة عوامل لبرل ، كالبوابات والاسوار، المناظر الطبيعية، المناطق الترفيهية،

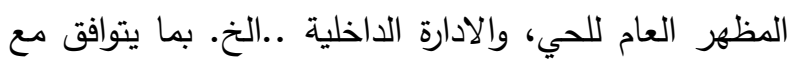
ابحاث كثيرة تم التعرض لها بالدراسة النظرية.

وبصورة عامة فقد أظهر النموذج المقترح امكانية واسعة في تقييم المجتمعات المسورة بقيم واضحة وبطريقة سلسة لا تتطلب كثير من الوقت والتحليل. كما تظهر تحليل

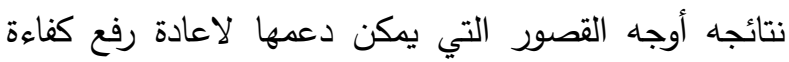


Vol. 40, No. 1. January 2021

22(7), 553-561 (2004)

[19] Marican, S.: Kaedah penyelidikan sains sosial. Petaling Jaya: Pearson (2005)

[20] KIM, SUK: THE GATED COMMUNITY:RESIDENTS' CRIME EXPERIENCE AND PERCEPTION OF SAFETY BEHIND GATES AND FENCES IN THE URBAN AREA. Ph.d, Texas A\&M University (2006)

[21] Blakely, E.J., Synder, M.G.: Separate Places: Crime and Security in Gated Communities. Washington:Urban Land Institute (1997)

[22] said, N.S., Martin, D.J.: The housing environment preference among housing consumers in Johor Bahru. In : 2nd International Conference on Technology Management, Business and Entrepreneurship. Melaka, Malaysia, pp.58-68 (2013)

[23] Tanulku, Basak: Gated communities: From "Self-Sufficient Towns", to "Active Urban Agents". Geoforum 43, 518-528 (2012)

[24] Yousry, Ahmed: The Privatization of Urban Development in Cairo: Lessons Learned from the Development Experience of Al Rehab Gated Community. In : the International Conference on Developing the New Urban Communities; Policies and Priorities, New Urban Communities Authority, Alexandria (2009)

[25] Shawish, Ayla: Gated communities and neighborhood livability in Doha. MS.c.,Qatar University, Doha,Qatar (2016)

[26] Cao, H.,: The governance of gated communities: International experience and the practice in China. Wuhan University Jourmal (Arts and Humanity) 70, 5-14 (2017)

[27] Salah, Nesma, Ayad, Hany: Why people choose gated communities: A casestudy of Alexandria metropolitan area. Alexandria Engineering Journal 57(4), 2743-2753 (2018)

[28] Güzey, Özlem: Neoliberal urbanism restructuring the city of Ankara: Gated
RESIDENTS (CASE STUDY: MERU HILLS, IPOH). In : Annual Serial Landmark International Conferences on Quality of Life, ASEAN-Turkey, vol. 222(2016), pp.747-754 (2015)

[10] Adriaanse, C.: Measuring Residential Satisfaction: A Residential Environmental Satisfaction Scale (Ress). Journal of Housing Build Environment 22, 287-304 (2007)

[11] Faggal, Ahmed: Gated Communities as an approach toward Sustainable Development. (Accessed 2012) Available at: DOI: 10.13140/RG.2.2.14813.20962

[12] Zadeh, LA.: Fuzzy sets. Inf Control 8, 338353 (1965)

[13] Wang, Wel-Ming, Lee, Amy, Chang, DingTsair: An integrated FDM-ANP evaluation model for sustainable development of housing community. Optimization Letters 4, 239-257 (2010)

[14] Eraqi, Ayman: Development a fuzzy model to predict the index of urban development priorities of the parties to the Egyptian city in partnership between the Cooperative Trinity. In : Urban Planning and Architecture Design for Sustainable Development, UPADSD, Lecc,Italy, pp.129-140 (2016)

[15] Chang, P., Wang, Y.: Fuzzy Delphi and BackPropagation Model for Sales Forecasting in Pcb Industry. Expert Systems with Applications 30(4), 715-726 (2006)

[16] Zainal, Z.: Case study as a research method. Jurnal Kemanusiaan 9 (2007)

[17] Thomas, A., Kalidindi, S., Ananthanarayanan, K.: Risk perception analysis of BOT road project participants in India. Journal of Construction Management and Economics 21(4), 393-407 (2003)

[18] Long, N., Ogunlana, S., Quang, T., Lam, K.: Large construction projects in developing countries: a case study from Vietnam. International Journal of Project Management 
[39] Adham, K.: Globalization, Neo-liberalism, and New Spaces of Capital in Cairo. TDSR 17(1), 19-32 (2005)

[40] Said, N.: Cairo behind the gates: studying the sensory configuration of Al-Rehab City. (Accessed 2013) Available at: http://ambiances.revues.org/252

[41] Chu, H.C., Hwang, G.J.: A Delphi-based approach to developing expert systems with the cooperation of multiple experts. Expert Syst. Appl. 34, 2826-2840 (2008)

[42] Wey, W.M., Wu, K.Y.: Interdependent urban renewal project selection under the consideration of resource constraints. Environ. Plan. B: Plan 35, 122-147 (2008)

[43] Wey, W.M., Wu, K.Y.: Using ANP priorities with goal programming in resource allocation in transportation portation. Math. Comput. Model. 46, 985-1000 (2007)

[44] Murray, T., Pipino, L., Van Gigch, J.: A pilot study of fuzzy set modification of Delphi. Human Systems Management 5(1), 76-80 (1985)

[45] Saaty, T.L., Vargas, L.G.: Diagnosis with dependent symptoms: Bayes theorem and the analytic hierarchy process. process. Oper. Res. 46(4), 491-502 (1998)

[46] Jaderi, F., Ibrahim, Z.Z., Jaafarzadeh, N., Abdullah, R., Shamsudin, M.N., Yavari, A.R., Nabavi, S.M.B.: Methodology for modeling of city sustainable development based on fuzzy logic: a practical case. Journal of Integrative Environmental Sciences 11(1), 71-91 (2014)

[47] Mert, Zeynep: Mert, Zeynep Gamze anFuzzy Modeling Approach Based on Property Location Quality for Grading Neighborhoo Level of Family Housing Units. Expert Systems with Applications 36, 3603-3613 (2009)

[48] Zayed, T., Halpin, D.: Quantitative Assessment for Piles Productivity Factors. J. Constr. Eng. Manage 130(3), 405-414 (2004) communities as a new life style in a suburban settlement. Cities 36, 93-106 (2014)

[29] Shamsudin, Zarina, Shamsudin, Shafiza, Zainal, Rozlin: Factors influencing resident's decision to reside in gated and guarded community. In : AIP Conference Proceedings, pp.1891, 020131 (2017) (2017)

عبد الله, سحر, الإبياري, ناهد: المجتمعات المغلقة كنموذج [30]

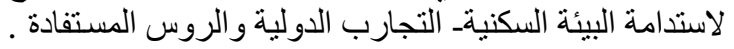

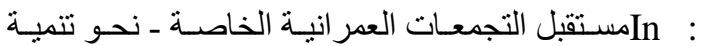

مستدامة, القاهرة (2013) - (2013)

[31] Atkinson, R., Blandy, S.,: Gated communities /privatopias. Encyclopedia of Human Geography, 297-301 (2009)

[32] Careford, John: Unlocking the Gates: Social Capital and Gated Communities. MS.c, University College London, London (2007)

[33] Zhaoa, Pengjun, Zhangb, Mengzhu: Informal suburbanization in Beijing: An investigation of informal gatedcommunities on the urban fringe. Habitat International 77, 130-142 (2018)

[34] Chapman, David: Analysis of determinants in neighborhood satisfaction between defended and defensible communities within the general urban housing environments. old dominion university,Ph.D (2007)

[35] Misun Hur, Hazel: Factors That Influence Residents' Satisfaction With Neighborhoods. Environment and Behavior 40(5), 619-635 (2008)

[36] Khalil, Ashraf: Egyptian dream takes shape in alien suburbs. Los Angeles Times 22 (2007)

[37] Denis, Eric: Cairo as Neo-Liberal Capital: From Walled City to Gated Community, inDiane Singeman and Paul Ammar. American University in Cairo Press, Cairo (2006)

[38] Craig, Geoffery: Building dreams or chasing a mirage? American Chamber of Commerce in Egypt Business Monthly. (February 2008) 
[52] Jayasree, K., Bharathi, M., Srilatha, A.: Application of Fuzzy Logic in Urban Planning For TIA. International Journal of Engineering Research \& Technology (IJERT) 2(3) (2013)

[53] Alkan-Bala, Havva, Üstüntaş, Taner: Modelling the Urban Interface by Using Fuzzy Logic. Journal of Building Construction and Planning Research 2, 59-73 (2014)

[54] Alwetaishi, M., Gadi, M., Issa, U.: Reliance of building energy in various climatic reions using multi criteria. International Journal of Sustinable Built Environment 6, 555-564 (2017)

[55] Al-rehabcity web-site Available at: http://www.alrehabcity.com
[49] Shang, H., Anumba, C., Bouchlaghem, D., Miles, J.: Distributed Construction Teams." "Assessment for Distributed Construction Teams. An Intelligent Risk Assessment for Eng., Constr. Archit. Manage. 12(4), 391-409 (2005)

[50] Ma, H., Deng, Z., Solvang, W.: An Online Approach for Distributor Benchmarking. Benchmarking Int. Journal 11(4), 385-402 (2004)

[51] Hashemi, Farhad, Sion, g: Extracting Urban Sustainability Indicators for Tehran Metropolis by Fuzzy Delphi Method. MiddleEast Journal of Scientific Research 22(9), 1411-1421 (2014) 